\title{
Software Defined IPv6 Network: A New Paradigm for Future Networking
}

\author{
Babu R. Dawadi ${ }^{1 a}$ ，Danda B. Rawat ${ }^{2}$ ，Shashidhar R. Joshi ${ }^{1}$ \\ ${ }^{1}$ Pulchowk Campus, Institute of Engineering, Tribhuvan University, Kathmandu, Nepal \\ ${ }^{2} \mathrm{CWiNs} / \mathrm{DSC}{ }^{2} \mathrm{Lab}$, Howard University, Washington D.C., USA \\ Corresponding author: ${ }^{a}$ baburd@ioe.edu.np
}

Received: Nov 11, 2018

Revised: Jan 27, 2019

Accepted: Feb 3, 2019

\begin{abstract}
Recent advancement in the field of Information and Communication Technology (ICT) has encouraged all stakeholders to move towards the new networking paradigm. Internet Protocol version 6 (IPv6) addressing, Software Defined Network (SDN) and Network Function Virtualization (NFV) are regarded as technologies for enhancing network efficiency and effectiveness. However, the technology migration becomes one of the central challenges for the stakeholders such as service providers, end users, and regulatory bodies. This is more challenging in case of developing countries due to lack of sufficient cost and skilled human resources. In this paper, we provide an overview and survey of SDN and IPv6 networking technologies, their benefits and future challenges. Then we introduce Software Defined IPv6 (SoDIP6) network as a next generation networking technologies and their unified approach of deployment over the Tier-3 ISPs of the developing nations that could help for speedy and smooth migration with optimized cost. The demonstrated superior features of SDN enabled IPv6 network from different perspectives with its contributions to green ICT are recognized as the networks of the future generation in the networking world.
\end{abstract}

Keywords: IPv6, SDN, SoDIP6, Service Providers, Migration Cost, CapEX/OpEX

\section{Introduction}

With the advancement on Information and Communication technologies (ICT), we have high volume of networking devices connected to internet that led to the emergence of Internet of Things (IoT). According to the gadget survey, within the last two decades, the number of internet users reached more than 3 billion and the mobile gadgets reached more than 7.2 billion. In other words, number of connected devices to the internet is increasing exponentially. Studies have already shown that the legacy IPv4 networks which dominate the current Internet - cannot handle the projected number of networked devices and applications. IPv6 has been alternative solution to handle the issues related to addressing of exponentially increasing networked devices. Furthermore, Software Defined Networking (SDN) is regarded as an emerging paradigm that provides better visibility and controllability with security to the network for better performance and efficiency. IPv6 helps improve the efficiency of internet protocol including addressing, security and routing, and the SDN helps improve the manageability of networks by using open protocols and SDN controller. Our 
goal is to study how migration of IPv4 based network to SDN enabled IPv6 (SoDIP6) could help get better efficiency with lower cost. Migration is a gradual process for which a proper strategy has to be developed considering the technology needs, customer demand, Capital Expenditure (CapEx), Operational Expenditure (OpEx) and traffic engineering perspectives towards smooth transitioning. The transition period may span longer during when the service providers should have to move on by following the best migration planning with optimum cost. Migration is to mean the upgrade of the existing network to make it operable with new technologies. Routers and the switches are the core components of the service network which are to be able to operate with the newer technologies. Either upgrade of the software/firmware version or device replacement is required with the investment on CapEx and OpEx for the service providers. Immediate service provisioning of newer technologies according to customer demand could not be viable for service providers. Hence, the customer profiling and prioritization is required to provide the new and upgraded service as per Service Level Agreement (SLA). For the fairly sustained network service providers, it is more important to develop the strategic plan for migration and estimate the cost incurs for total migration of network together with the guarantee of uninterrupted service to their customers. In this paper, we study the network migration from legacy IPv4 network to SDN enabled IPv6 with an aim of enhancing the overall performance of the network and minimizing the overall cost.

The rest of this paper is organized as follows. Section 2 summarizes the major challenges of network migrations. Section 3 provides the brief literature review and common issues on migration to new networking paradigms. Section 4 highlights the network migration considerations for service providers. The cost/benefit and return of investment analysis for migration to SoDIP6 network is presented in section 5 and the paper is concluded in section 6 .

\section{Challenges of Network Migration}

Following are challenges to be addressed by the stakeholders during their network migration:

- Cost of network device migration/replacement: Sometimes it is hard to any fairly sustained service providers and enterprises to migrate their networking system into operable latest technologies due to lack of sufficient fund especially in developing countries. The budget limit is also the major factor that might delays the migration. Preliminarily, it is required to identify the status of existing network equipment whether it can be upgraded to or it must be completely replaced with new equipment. This depends upon the device performance as well as availability of latest software/firmware that is able to operate with IPv6 over SDN. The cost of upgrades or replacement has to be estimated with phase wise migration planning considering the ceiling of the budget that is available for every fiscal year. Basically, cost involves in the several dimensions not only to the device upgrades or replacement but also to the installation, testing and development of skilled human resources who operate and maintain the system.

- Availability of stable networking \& server applications: The migration depends upon the readiness of network and server applications. The readiness status of hardware, operating system, client and server applications, end user equipment, security systems and many more are to be evaluated based on its functionality for the better continuity of services.

- Energy efficiency and security during and after the migration: With the expansion of the network size, hardware and software, energy bill is increasing every year. Optimization on 
energy consumption by network equipment focusing to green computing in SoDIP6 network operation is also a major concern for the service providers. Similarly, security is another major concern to guarantee that whether the new technologies after implementation or during implementation is well secure or not. It is obvious that implementation of new technologies and integrated it with the existing legacy one during the transition can create security risk. The firewalls, servers and other applications security should also be able to function well with new technologies.

- Guarantee of uninterrupted service to the customers during migration: This is one of the major concerns in many developing countries that delays the network migration to SDN enabled IPv6 network. Service providers are always in fear of service interruption while approaching the new technology in the implementation.

Although IPv6 deployment strategies with the possible approaches of migration have been identified, SDN deployments over the Internet Service Providers (ISP) and Telecom Service Providers (Telcos) are in the premature stage. This encourages us to have research on the strategic deployment of SDN enabled IPv6 (SoDIP6) in the service providers' network with the estimation of cost involve during the migration by taking the priority of customers and the service level agreements. In this paper, we study the technical and economic part of the legacy network migration to SoDIP6 network so that service providers (of developing countries) would no longer be in trouble/crisis of their infrastructure transforming to adaptable new technologies including SDN enabled IPv6.

\section{Literature Review on SDN and IPv6 Transition}

IPv6 addressing mechanisms, SDN and Cloud Computing are major inventions that the network and cloud service providers are emerging to use for robust service and better efficiency. IPv6 is the network layer protocol invented to avoid the current issues of IPv4 addressing. SDN has been established to avoid the issues of existing network towards the highly manageable and programmable network by detaching the control plane from each device into the centralized one so called the controller. SDN paradigm is popularly recognized in the data center while its implementation practices in the service provider networks are in progress. IPv6 on the other hand is becoming compulsion for the service provider to migrate their network at the earliest to solve the issues with increasing number of connected devices. Combining the IPv6 and SDN is expected to ease the process of transitioning from legacy to SoDIP6 and improve the overall network performance and efficiency.

\subsection{SDN Enabled IPv6 (SoDIP6) Network}

SDN and the IPv6 are interrelated technologies where IPv6 operate on the network layer and SDN deals with the management of the networking operations. IPv6 promises to add scalability and better quality of service while SDN promises to increase the infrastructure flexibility with the ondemand provisioning of network resources and integrated into overall cloud orchestration.

"The future networking infrastructure fully IPv6 operable and controlled/managed by the SDN controller is recognized as a SoDIP6 network".

Migration to IPv6 network is evaluated by the readiness of existing network as IPv6 ready. Migration to SDN catalyzes the IPv6 network migration such that the SDN should be IPv6 based 
not only the IPv6 ready. On the other hand, the ongoing research enforces that the migrated IPv6 network should be controlled via SDN controller.

\subsubsection{IPv4 vs. IPv6}

IPv6 has the major features as classless addressing mechanism, standardized multicast addressing, address auto configuration (SLAAC) [27], new and optimized header format with fixed 40 bytes header length, special header field like flow-level for quality of service, inbuilt security with IPSEC framework, improvement on ICMPv6 [5] with the incorporation of ARP and IGMP like protocols, efficient and hierarchical routing infrastructure, oversize packet fragmentation by the IPv6 host itself instead of router and many more. The main task of router is the optimal path finding to route the packet to proper destination. But sometimes IPv4 routers are overloaded due to fragmentation of oversized packets by itself. Hence IPv4 has special header fields (identification, flag \& fragmentation offset) to handle fragmented packets. But IPv6 router never fragments the packet itself. Instead it generates the ICMPv6 packet too big error message to the source so that source host itself is liable to fragment the packets below MTU size [15]. The packet header size in IPv4 is variable changing form 20-60 bytes where the 8 bytes (source and destination address) are not required by the router to be processed. Every time the router requires viewing the header size due to variability nature of IPv4 packet header length. The packet header is efficiently modified in IPv6 and it is fixed 40 bytes in which 32 bytes (source and destination address) are not required for the router to be processed. IPv6 header has special header field called flow level in addition with modified header field traffic class to handle the traffic which requires special handling for better quality of service. IPv6 header with its extension headers are managed into daisy chained fashions that ensure extensibility features of IPv6. The built in ESP (Encapsulating Security Payload) and AH (Authentication) extension headers and protocols defined under IPSEC framework ensures better security in IPv6 capable networks.

\subsubsection{IPv6 Transition Methods}

It has already been two decades that the specification of IPv6 was developed in 1998. The IPv4 address pool was first exhausted in Asia region in April 2011 announced by Asia Pacific Network Operation Center (APNIC) while the exhaustion in other Regional Internet Registries (RIR) took some years and exhausted before the projection date. RIR for Latin America (LACNIC) ran out of IPv4 addresses on June 2014. Similarly, RIR for North America (ARIN) and RIR for Africa (AFriNIC) got exhausted on September 2015 and April 2017 respectively [26]. However, some countries have already planned for migration and their service providers are gradually migrating their network into IPv6, most of the countries are lacking behind the IPv6 implementation. Google measurement of global IPv6 penetration status till January 2018 is almost $18 \%$ as depicted in Fig. 1. World's IPv6 network capability in an average is only crossing $17 \%$, while major twenty countries IPv6 capabilities plotted from APNIC lab is shown in Fig. 2. It shows that the world's IPv6 migration status is fairly progressive. Fig. 2 indicates that the IPv6 adoption rate of developed countries is comparatively high but the rate of adoption in the developing countries of Asia and the African region is as low as below $1 \%$.

Three migration approaches i) Tunneling, ii) Dual Stack and iii) Translation have been identified for the smooth transitioning of current IPv4 operable network into IPv6 networks. Tunneling is the short-term solution that service provider could use for IPv6 services over IPv4 networks. Dual stack approach is the main task that service providers should move into it so that every network 
device can process both packets in its operation. Whether to use translation mechanisms depends on the outside network's interconnection status. At the final stage of the migration, translation approach is preferable once the current network is completely migrated into IPv6 while the limitedly interconnecting outside networks are still operating in IPv4. Moving the network into dual stack involves high cost for the service providers because each and every device from core network to Customer Premise Equipment (CPE) should have to have both IPv6 and IPv4 capable. This option is hardly achievable for developing countries where budget is always a constraint.

Dawadi et al. [6] have elaborated the different migration strategies for service providers by considering the situations in which migration strategies depends on the existing network status of the service providers. Benchmarking the networking equipment before transforming to IPv6 network is highly preferable. The option should ensure the business continuity with a plan of migration preparation, design and build the coexistence network and finally turn off the IPv4 network. Main et al. [13] has measured the organizational readiness factors of migrating to IPv6 network by taking the structured interview with different stakeholders such as IT experts, Banks, ISPs, and Academia etc. However, the core network is IPv6 ready, the organizations are not able to migrate their network due to other several factors such as requirement of migration (deployment) planning, equipment costs, technical expertise of the employees, etc. Fig. 3 shows the rate of increase of overall data/internet access rate of Nepal indicating that the overall data/internet penetration rate was increased from $2.63 \%$ at 2009 to $63.17 \%$ at Dec. 2017 . It shows that the internet penetration since 2009 to 2017 is linearly increasing. However, Considering only to fixed broadband, current broadband penetration rate of Nepal is only $10.50 \%$ and the mobile broadband $(3 \mathrm{G} / 4 \mathrm{G} / \mathrm{EVDO})$ is $36.81 \%$.

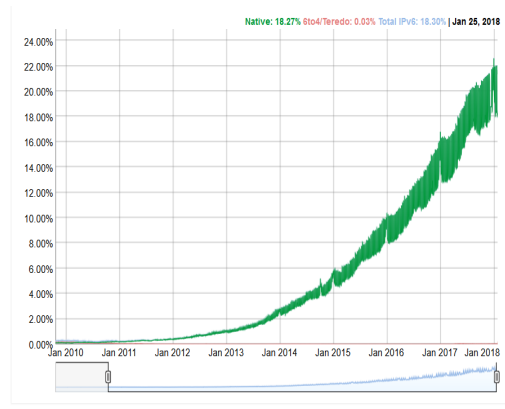

Fig. 1: IPv6 adoption statistics by Google

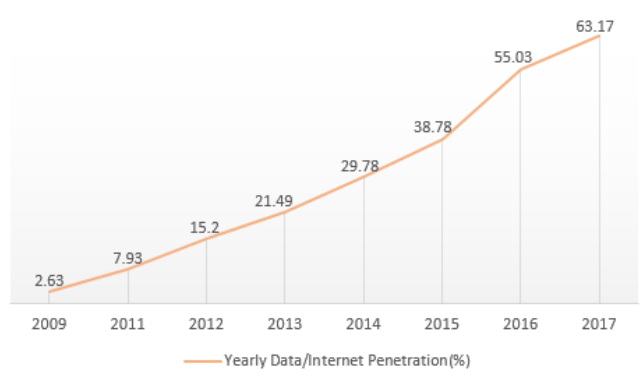

Fig. 3: Internet penetration rate of Nepal

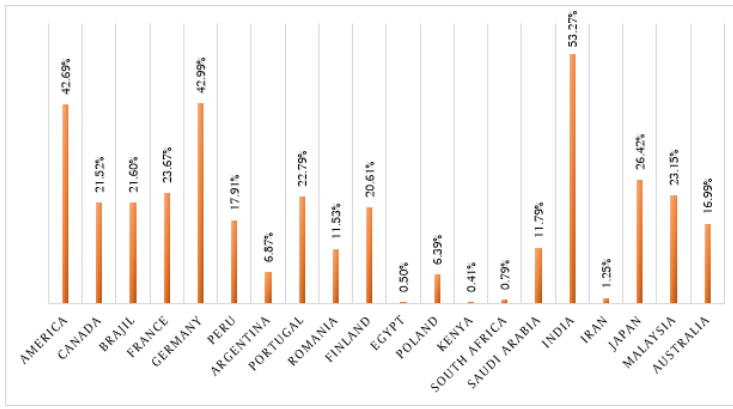

Fig. 2: IPv6 adoption status of selected twenty countries

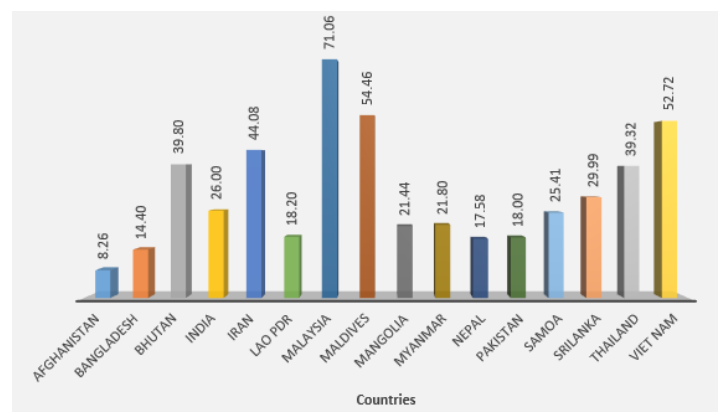

Fig. 4: Developing countries internet access status 
The individual internet user's rate of developing and least developed countries by $2016^{1}$ in Fig. 4 shows that there are lots of rooms for the growth of internet users globally. This also shows that service providers of developing countries are quite behind the IPv6 migration and so SDN too. To achieve the growth as expected, it is worth important to expand the networking infrastructure by the service providers. Due to the shortage of IPv4 address, infrastructure and service expansion with better quality of service for service providers is viable only with IPv6, while service providers can maximize efficiency, scalability, flexibility and manageability of the expanded IPv6 network and growth of internet users with the migration to SDN.

Adoption of IPv6 in real practice not only depends on the status of individual organization but also on what others is doing with their interconnected networks [17]. The worldwide distributed internet architecture belongs to several organizations such as service providers, academia, government, industries etc. Individual organizations depend on the status of interconnecting organizations that leads to the speed of migration in the chain. Different methods are in practice considering the progress of migration. Table 1 presents a comparative study of outline of the different transition methods with their features so that service providers can have their own choice of implementations.

With the availability of different transition methods, which method will be suitable to service providers depend on its existing network status. If a service provider doesn't want to migrate to IPv6, it can continue following the NAT44 and recursive NAT (NAT444) approach only in its network scope. Fig. 5 shows the transition path for service providers to migrate their current network into IPv6 only operable network. Once suitable migration approaches have been identified, service providers can follow the steps with optimum planning for migration.

\subsubsection{SDN and its Migration}

SDN provides a high speed manageable network and regarded as adaptable and directly programmable next generation network paradigm $[20,21]$ with the following features [11].

Table 1: Selected IPv6 transition methods \& their features

\begin{tabular}{lllllll}
\hline \multicolumn{1}{c}{ Methods/Features } & $\begin{array}{c}\text { IPv6 } \\
\text { support }\end{array}$ & $\begin{array}{c}\text { IPv4 De- } \\
\text { pletion }\end{array}$ & $\begin{array}{c}\text { Co-Ex- } \\
\text { istence } \\
\text { with } \\
\text { IPv6 }\end{array}$ & $\begin{array}{c}\text { Customer } \\
\text { Premise } \\
\text { Equipment } \\
\text { Change }\end{array}$ & $\begin{array}{c}\text { Operational } \\
\text { Complexity }\end{array}$ & $\begin{array}{c}\text { Investment } \\
\text { Cost }\end{array}$ \\
\hline $\begin{array}{l}\text { CG NAT (DS with Pri- } \\
\text { vate IPv4 + GUA) [29] }\end{array}$ & Yes & Yes & Yes & No & Yes & Low \\
NAT44/NAT444 [2] & No & Yes & No & No & Medium & Low \\
464XLAT [14] & Yes & Yes & Yes & No & High & Medium \\
6RD [28] & No & Yes & Yes & Yes & Low & Low \\
Dual Stack (DS) & Yes & Yes & Yes & Yes & Low & High \\
[18] & Yes & Yes & Yes & Yes & High & Medium \\
DS Lite [12] & No & Yes & Yes & Yes & Medium & Low \\
6to4 [3] & Yes & Yes & Yes & Yes & Low & Low \\
MAP-E/MAP-T [4] & & & & & & \\
\hline
\end{tabular}

1 ITU IDI Index-2016: http://www.itu.int/net4/ITU-D/idi/2016/ 


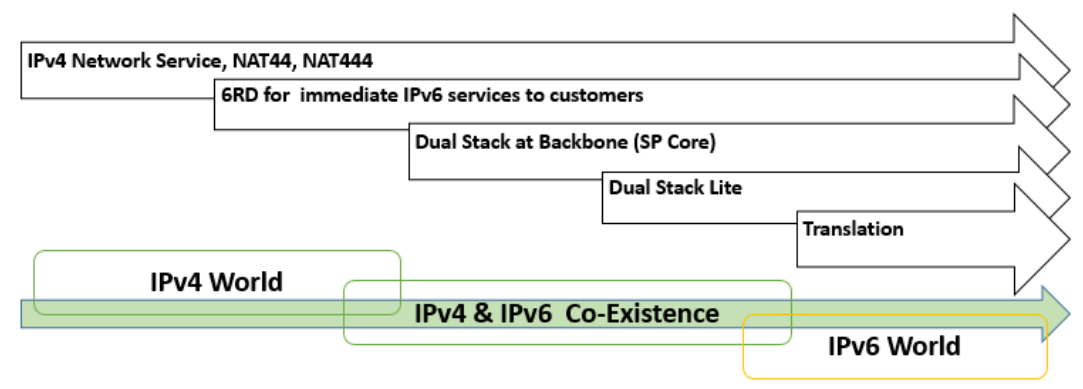

Fig 5: Path of transitioning to IPv6 network for service providers

- Separation of control and data plane: The control plane of individual devices is removed and centralized into the SDN controller. This implies that these two planes reside independently in the network with close communication. Data plane of the network devices simply act as a packet forwarding passive elements based on the decision made by the controller. Logically centralizing the controller offers several additional benefits like better support to traffic engineering, virtualization, security enhancement, consistent policy implementation with less error prone to modify network policies, better surveillance system that automatically react to spurious changes of the network state keeping the high-level policies intact. Additionally, it enables the developer to implement sophisticated network services and applications.

- Flow based: Unlike the legacy network which performed destination-based decision, SDN controller makes the flow based forwarding decisions. A flow is considered as the set of packets having identical service policies flowing from source to destination.

- Controller functionality: The SDN controller is a management software platform that runs on top of commodity server technology and facilitates provisioning essential resources to the programming of forwarding devices based on a logically centralized, abstract network view.

- Programmable network: Behavior of network devices and flow control is handled by a software program running on the controller. Network programmability is the fundamental characteristics of SDN that allow network engineer to reprogram the network infrastructure on the fly.

- Open interface: The presence of open Application Programming Interface (API) enables standards to define open interface.

- Abstraction: SDN applications are abstracted from its underlying network technologies to support equipment with vendor neutrality, technologies and enable control plane to support varieties of applications,

Fig. 6 depicts the situation of legacy networking devices where control and data plane of each device is integrated with itself making the network complex in management and configuration. While Fig. 7 depicts the future network situation where the control plane from each device is removed and moved to a single controller. SDN enabled network nodes are controlled by network operating system that collects information in the controller.

The functional architecture of SDN is hence consists of three layers as infrastructure, controller and applications. The communications among the layers are defined by the use of API like north bound and south bound APIs while communication among the controller in the distributed environment shall be achieved through east/west bound APIs. Fig. 8 depicts the overall functional architecture of SDN. The control plane software shall run on general purpose commodity hardware so called SDN controller. SDN enabled network nodes are controlled by network operating system that 
collects information in the controller. Every SDN enabled switch maintained flow tables in its data plane. The basic algorithm that how the data plane contacts with controller and forwards the traffic based on its Flow Table is shown in Fig. 9

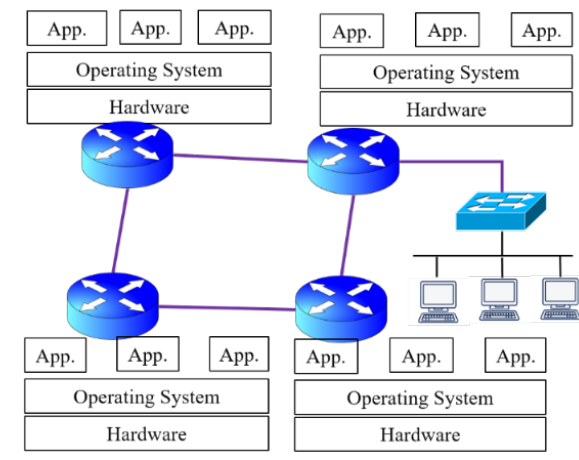

Fig. 6: Legacy network structure

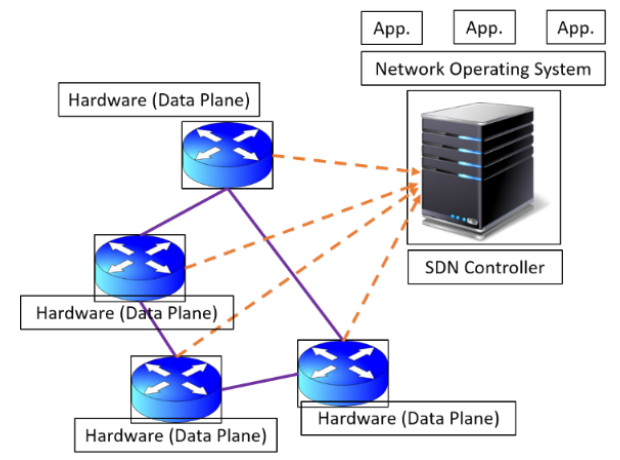

Fig. 7: Typical SDN structure

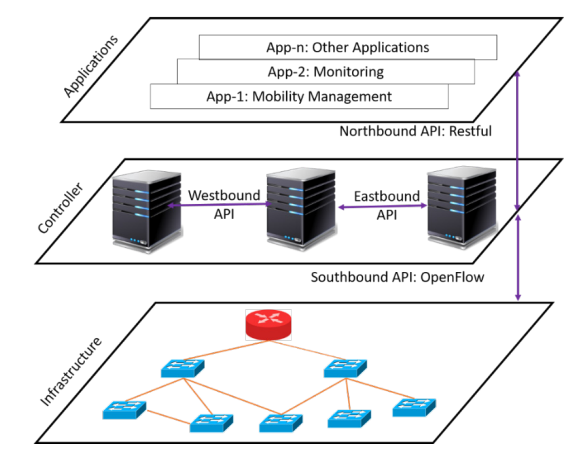

Fig. 8: SDN functional architecture

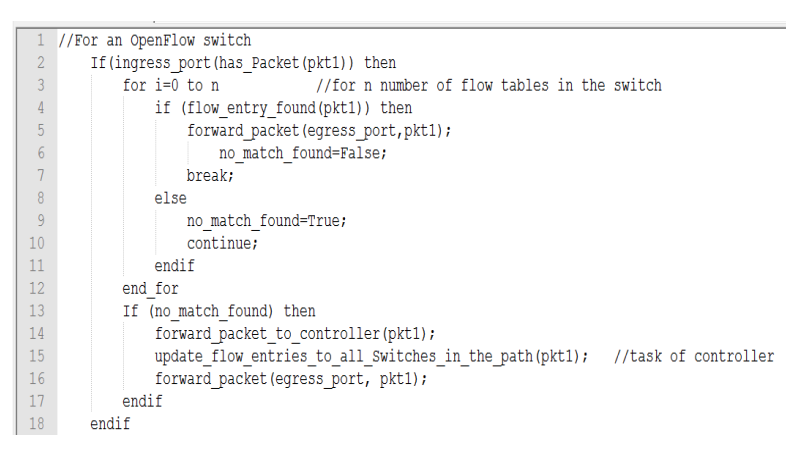

Fig. 9: Functions of OpenFlow switch

Basically the OpenFlow switch checks the flow entries of incoming packet into its flow table and forwards the packet if entry is matched; otherwise the switch sends the packet header to the controller to get update into its flow tables. The controller updates flow tables of the entire switches towards the path of the packet's destination.

Migration to SDN enabled network is a challenging task same like that of migration to IPv6 enabled network. With the ongoing research and development, network operators world-wide mostly concerned with the cost of migration, performance, service availability, management and security. Legacy to SDN migration approaches have three categories with preliminary steps in migration, these are:

- Legacy to Greenfield: This approach simply replaces the legacy IPv4 compatible network devices with OpenFlow enabled SDN devices. It is simple but for the real life implementation and business purpose, this approach is not feasible. Gradual replacement of equipment with the service continuity is to be considered.

- Legacy to Mixed Network: In this approach, the targeted network consists of both legacy routers/switches and the SDN enabled routers/switches. This proposes the coexistence of network in which interoperability and communication among SDN and non-SDN devices are to be established. 
- Legacy to Hybrid Network: The target network consists of hybrid devices which are well capable to communicate with both the OpenFlow and traditional router/switches. Hence the hybrid devices interface with both OpenFlow controllers and legacy control plane.

\section{Network Migration Considerations}

While the basic things that service providers need to consider for SDN \& IPv6 network migrations are:

- Network Inventory Management \& Identify Network Status: maintaining the network equipment detail information like performance values, life cycle, physical connectivity \& topology details are the important part of any small and large organizations. The main task is to benchmark the current network devices with respects to its firmware versions and upgradability scenarios. This helps to plan for the migration with the requirement of cost involve during the migration, security consideration, replacement, upgrade and maintenance of the network equipment.

- Identify and prioritize core requirements of the network to be migrated: it is the requirements identification process of the target network or Software Defined IPv6 Network which looks to be after migration. It helps to design the network as per the requirements.

- Prepare Annual Technology Budget: replacement or upgrades of equipment incurs cost. Considering the organizational OpEX, it is required to prepare annual expenditure towards the technology upgrades and pilot test on any new technologies.

- Prepare a starting network for the pilot test: either in the virtual or real environment, the best way is to create a pilot network for network functioning and inter-operability test.

- Customer Profiling and the Service Level Agreement: for a service provider, there might be different network/internet services like internet bandwidth, content, cloud services, MPLS, VPN provided to the customers of different kinds like government offices, banks, companies, academia and the normal public. It is required to prioritize the customers who is in need of immediate services to be executed over IPv6 network and service of SDN.

- Phase-wise network migration: SLA guide the priority of customer and helps for phase wise migration so that service provider shall decide about which network path is to be migrated first. It is required to consider the customer demand, technology readiness, human resource readiness and more for the network migration. Hence it is better to migrate the network into phase by defining the transition period so that possible service interruption shall be avoided.

- Validate the migration: Based on the predefined requirements, the migrated network is to be validated.

\section{Cost/Benefit and Return on Investment Analysis of Migration}

SDN could help reduce the CapEX/OpEX of the organizations [24] which encourages the service providers to search for the better options and attraction towards SDN. Besides the implementation challenges [25], SDN is proven technology towards efficient network management that it solves those existing issues and create highly flexible, programmable, scalable, modular, open interface and abstraction-based networks [20, 23]. The major stakeholders like Stanford Campus Network, Google Data Center, NTT communication and AT \& T [1, 10, 19, 20] have already deployed the prototypes and implementing SDN with the migration in a phase. This encourages other service providers to decide for the migration.

This section presents some recent works studies on migration cost/benefit and return on investment 
analysis of network migration. In our previous work [7], we present the joint cost estimation approach for unified migration and we verify that unified migration to SDN enabled IPv6 network would reduce the total cost of migration. With the increase of network infrastructure, the energy bill for operators increases. Legacy IPv4 networking is energy hungry in which network switch and routers operate with peak energy consumption even in the idle condition leading to wastage of energy and constitutes higher operational cost. The National Institute of Standard Technology (NIST) report elaborates the IPv6 economic impact assessment to predict the total cost of network migration to IPv6 [16]. It is predicted that the provisioned IPv6 adoption cost for the ISPs is US\$2.6 million and internet cost is US $\$ 1.8$ million for the year 2018 while this cost would reduce almost to zero in the year 2022. The percentage of total transition cost is identified to be divided into categories like software upgrade cost (19.3\%), Testing (18.3\%), Installation (10.7\%), Maintenance $(12.0 \%) \&$ human resources as training cost (33.0\%). This implies that the training or labor cost is comparatively high. Most of the IPv6 transition approaches require CPE change for the end users. CPE change could be a major investment for service providers or either it has to bear by customer. Looking into the benefits and return on investment, Table 2 presents the overview of IPv6 and SDN benefits based on the study presented in [16] and [30].

Huge investment on infrastructure and technology migration without the guarantee of return on investment is difficult for fairly sustained service providers. The benefits of migrating to new technologies like SDN and IPv6 is more qualitative in nature. Several researches verified that both the IPv6 and SDN technologies are far better than the existing legacy IPv4 network. The continuity of existing legacy IPv4 network is possible only for certain period either purchasing the NAT device like CGN and/or purchase the available IPv4 public address from the trading market. CGN approach could exists until other service networks run the IPv4 but due to depletion of public IPv4 address, its availability in the market would be limited with the higher cost. Table 3 shows the costs and its associated revenue loss due to continuing the legacy network.

Table 2: Benefits of transitioning to SoDIP6 network

\begin{tabular}{lll}
\hline Benefits & Factors of realizing the benefits & Applies to \\
\hline Address space & - & $\begin{array}{l}\text { The larger IPv6 address space opens opportunities for scalable } \\
\text { network design, growth of end users and smart devices. }\end{array}$ \\
& $\begin{array}{l}\text { The existing NAT solutions and its associated problems will be } \\
\text { avoided. }\end{array}$
\end{tabular}

Network - Flexible and horizontally integrated programmable network IPv6 and Administration decreases the overall network administration cost. Removal of SDN cost NAT/CGN reduces the network operation cost and significantly save the annual revenue with increased efficiency of network in the large scale. NIST report mentions that removal of NAT leads to an annual cost reduction of US\$ 1.8 million in user network installation and testing.

Quality of - Implementation of flow-level field of IPv6 header introduces service QoS aware networking operations and services. Better quality IPv6 increases the reliability and so encourage organizations to move to digital packet based communication like VoIP that may lead to savings on $20 \%$ or more in the annual telephony expenditure. 


\begin{tabular}{lll}
\hline Benefits & Factors of realizing the benefits & Applies to \\
\hline Security & $\begin{array}{l}\text { Security threats and worldwide cybercrime cost people and } \\
\text { businesses, a loss of more than US \$200 billion per year }{ }^{2} \text {. The Built- } \\
\text { in IPSec framework in IPv6 implementation can significantly } \\
\text { improve the network security. Similarly, Programmable Network } \\
\text { (SDN) enables to apply different security models to control } \\
\text { modern security breaches. }\end{array}$ \\
\hline
\end{tabular}

Table 3: IPv6 network transition cost analysis [9]

\begin{tabular}{|c|c|c|}
\hline Description & Associated costs in USD & Remarks \\
\hline $\begin{array}{l}\text { CGN } \\
\text { operation per } \\
10,000 \text { users }\end{array}$ & $\begin{array}{l}\text { - Capital Cost (purchase Hardware, } \\
\text { software and logging system) - } \\
\$ 90,000 \\
\text { - } \text { Operation expenses }-\$ 10,000 \\
\text { - Yearly depreciation on CapEX - } \\
\quad \$ 18000 \text { \& OpEX - } \$ 10,000 \\
\text { - } \text { Customer Support - } \$ 13,040\end{array}$ & $\begin{array}{l}\text { Total yearly revenue loss of US } \$ 20,800 \text {. } \\
\text { Hence the CGN operation cost is } \$ 30 \text { per } \\
\text { user per year. }\end{array}$ \\
\hline $\begin{array}{l}\text { IPv4 address } \\
\text { trading }\end{array}$ & $\begin{array}{l}\text { - } \$ 16-20 \text { per address in the year } 2016 \\
\text { - Higher cost (even }>\$ 100) \text { per address } \\
\text { since from the year } 2017 \text { due to } \\
\text { complete exhaustion of IPv4 address } \\
\text { in IANA. }\end{array}$ & $\begin{array}{l}\text { Due to exhaustion of IPv } 4 \text { address space } \\
\text { in the IANA pool. IPv4 address trading } \\
\text { in the market increases the cost of } \\
\text { purchasing IPv4 address from third party. }\end{array}$ \\
\hline $\begin{array}{l}\text { Enable Dual } \\
\text { Stack in the } \\
\text { network }\end{array}$ & $\begin{array}{l}\text { - The cost to run dual stack (IPv4 and } \\
\text { IPv6 both) for ISP is } \$ 7.50 \text { per user } \\
\text { per year. } \\
\text { - Deployment cost is } \$ 25 \text { per user }\end{array}$ & $\begin{array}{l}\text { This indicates that the dual network } \\
\text { deployment is comparatively costly. } \\
\text { Reduction on this cost is possible if } \\
\text { service provider limits the duration of } \\
\text { dual stack operation so that after disabling } \\
\text { the IPv4 stack will reduces those costs. }\end{array}$ \\
\hline
\end{tabular}

Additionally, we experimentally verified that SoDIP6 network is energy efficient and has significant annual cost saving for the service providers [8]. Rawat et al. [22] mentioned that the increasing network size and continuous service support increase the energy consumption by the network devices for which the companies pay a significant percentage of their revenue to power their network infrastructures. He has elaborated and evaluated different approaches for energy efficiency through SDN implementation in which the reduction of energy bill, optimal network resource utilization and proper placement of SDN devices for energy efficiency are the concerns need to be considered while in the migration planning.

\section{Conclusion and Future Works}

Due to the limitation of IPv4 address space, expansion to support growing number of connected devices which are uniquely identifiable globally can be achieved by using IPv6. To maximize efficiency, scalability, flexibility and manageability of the expanded network and growth of internet users, service providers need to migrate their networks to SDNs enabled IPv6 network. Developing countries with low internet penetration rate require optimum planning for migrating traditional network to emerging network. Traditional network cannot meet the exponential growth

2 https://www.cbsnews.com/news/id-theft-lucrative-for-web-crooks/ 
of users because of its static parameters that cannot be changed on the fly. Additionally, SDN provides better visibility, programmability and scalability of the network and IPv6 supports a large number of devices to identify uniquely in the global scale. SDN enabled IPv6 network is more robust and energy efficient so that organizational operation cost can be reduced. This paper has studied the advantages of migrating the legacy network to SDN enabled IPv6 network for service providers. The presented study is expected to be helpful for the service providers to optimally migrate their network to emerging network like SDN and IPv6. Adaptations to next generation networking system has better market growth for service providers and meet the requirements of future generations. Besides this, intelligent approach of network device status identification for migration, applications and protocol supports, security, quality of service and in overall the readiness measurement with the implementations are the major challenges to be considered in an ongoing future research of SoDIP6 network migration.

Acknowledgment: This research is supported by NTNU (Norwegian University of Science and Technology) under Sustainable Engineering Education Project (SEEP) and financed by the Energy and Petroleum Program (EnPe) in NORAD (Norwegian Agency for Development Cooperation). Additional funding support has been granted to us from Nepal Academy of Science and Technology (NAST).

\section{References}

[1] Babiker H, Nikolova I and Chittimaneni KK (2017), Deploying IPv6 in the Google Enterprise Network: Lessons Learned, https://www.usenix.org/legacy/events/lisa11/tech/full_papers/ Babiker. pdf, accessed November 2017.

[2] Bagnulo M, Matthews P and Van Beijnum I (2011), Stateful NAT64: Network Address and Protocol Translation from IPv6 Clients to IPv4 Servers, IETF - RFC6146, ISSN: 2070-1721.

[3] Carpente B and Moore K (2001), Connection of IPv6 Domains via IPv4 Cloud. RFC 3056.

[4] Congxiao B and Xing L (2013), IVI/MAP-T/MAP-E: Unified IPv4/IPv6 Stateless Translation and Encapsulation Technologies, In ZTE Communications, 11(3): 51-55.

[5] Conta A and Gupta M (2006), Internet control message protocol (icmpv6) for the internet protocol version 6 (ipv6) specification, $R F C 4443$.

[6] Dawadi BR, Joshi SR and Khanal AR (2015), Service Provider IPv4 to IPv6 Network Migration Strategies, Journal of Emerging Trends in Computing and Information Science, 6(10): 565-572, ISSN: 2079-8407.

[7] Dawadi BR, Rawat DB, Joshi SR and Keitsch MM (2018), Joint Cost Estimation Approach for Service Provider Legacy Network Migration to Unified Software Defined IPv6 Network, IEEE 4th International Conference on Collaboration and Internet Computing (CIC), Philadelphia, PA, USA, 372-379. doi: 10.1109/CIC.2018.00056.

[8] Dawadi BR, Rawat DB, Joshi SR and Keitsch MM (2019), Recommendations for energy efficient SoDIP6 network deployment at the early stage rural ICT expansion Nepal, Proc. of the 2019 IEEE International conference on communications and networking", Feb 18-21, Hunolulu, USA (accepted for presentation \& proc. publication).

[9] Howard L and Cable TW (2013), Internet Access Pricing in a Post-IPv4 Runout World. http:// www.wleecoyote.com/documents/TCOofCGN.pdf, accessed March 2018, accessed July 2018

[10] Kobayashi M et al. (2014), Maturing of OpenFlow and software-defined networking through deployments. Computer Networks, 61: 151-175.

[11] Kreutz D et al. (2015), Software Defined Networking: A comprehensive Survey, Proceedings of 
the IEEE, 103(1): 14-76.

[12] Lee Y, Maglione R, Williams C, Jacquenet C and Boucadair M (2013), Deployment considerations for dual-stack lite, $R F C 6908$.

[13] Main A, Zakaria NA and Yusof R (2015), Organisation Readiness Factors towards IPv6 Migration: Expert Review. Procedia-Social and Behavioral Sciences, 195: 1882-1889.

[14] Mawatari M, Byrne C and Kawashima M (2013), 464XLAT: Combination of stateful and stateless translation, RFC6877.

[15] McCann J and Deering S (1996), Path MTU Discovery for IP Version 6, RFC1981.

[16] Michael PG (2005), IPv6 Economic Impact Assessment, NIST Report-USA.

[17] Nikkhah M (2016), Maintaining the progress of IPv6 adoption, Computer Networks, 102: 50-69

[18] Nordmark E and Gilligan R (2005), Basic IPv6 Transition Mechanisms, RFC4213.

[19] ON.LAB White Paper, Driving SDN Adoption in Service Provider Networks, http://onosproject. org/wp-content/uploads/2014/11/Whitepaper-Service-Provider-SDN-final.pdf, accessed June 2018.

[20] ONF Solution Brief (2014), SDN Migration Considerations and Use Cases, ONF TR - 506, https://www.opennetworking.org/images/stories/downloads/sdn-resources/solution-briefs/sbsdn-migration-use-cases.pdf, accessed July 2018.

[21] ONF White Paper (2012), Software-defined networking: The new norm for networks, Palo Alto, $C A$, https://www.opennetworking.org/images/stories/downloads/sdn-resources/white-papers/ wp-sdn-newnorm.pdf, accessed Oct. 2018.

[22] Rawat DB and Reddy SR (2016), Software Defined Networking Architecture, Security and Energy Efficiency: A Survey, IEEE Communications Surveys \& Tutorials, 19(1):325-346, DOI 10.1109/ COMST.2016.2618874.

[23] Raza MH, Shyamala CS, Nafarieh A and Robertson B (2014), A Comparison of Software Defined Network (SDN) Implementation Strategies, Procedia Computer Science, 32: 1050-1055.

[24] Rizvi SN, Raumer D, Wohlfart F and Carle G (2015), Towards carrier grade SDNs, Computer Networks, 92: 218-226.

[25] Sakir S et al. (2013), Are we ready for SDN? Implementation challenges for software-defined networks, IEEE Communications Magazine, 51(7): 36-43.

[26] State of IPv6 Deployment (2017), https://www.internetsociety.org/resources/doc/2017/state-ofipv6-deployment-2017, accessed October 2017.

[27] Thomson S and Narten T (1998), IPv6 Stateless Address Auto-configuration. RFC2462.

[28] Townsley W and Troan O (2010), IPv6 Rapid Deployment on IPv4 Infrastructures (6rd), RFC 5969.

[29] Ungerman J (2012), Carrier Grade NAT: IPv4 Exhaustion and IPv6 Transition in Internet, Cisco CCIE\#6167,https://www.cisco.com/c/dam/assets/global/YU/expo2012/pdfs/tranzicija_prema_ ipv6.pdf, accessed Jan. 2018.

[30] US IPv6 Task force. Technical and Economic Assessment of Internet Protocol Version 6 (IPv6), https://www.ntia.doc.gov/legacy/ntiahome/ntiageneral/ipv6/final/IPv6final2.htm, accessed March 2018.

[31] Worldwide IPv6 deployment status, https://stats.labs.apnic.net/ipv6, accessed Jan 2018. 